11,12

\title{
Термодинамические и магнитные свойства двумерной анизотропной модели Изинга с конкурирующими взаимодействиями
}

\author{
(C) А.К. Муртазаев, Ж.Г. Ибаев
}

Институт фозики им. Х.И. Амирханова ДагНЦ РАН, Махачкала, Россия

Дагестанский государственный Университет,

Махачкала, Россия

๑ E-mail: ibaev77@mail.ru

Поступила в Редакцию 22 апреля 2019 г.

В окончательной редакции 22 апреля 2019 г.

Принята к публикации 26 апреля 2019 г.

\begin{abstract}
Проведены исследования двумерной анизотропной модели Изинга с конкурирующими взаимодействиями на квадратной решетке методами Монте-Карло с помощью алгоритма Ванга-Ландау. Рассчитаны температурные зависимости основных термодинамических и магнитных параметров. Показаны отличительные особенности температурных зависимостей этих параметров при разных значениях конкурирующего взаимодействия. Построены графики зависимости волнового числа модулированных структур от соотношения параметров обменных взаимодействий. Построена фазовая диаграмма модели. Интерполируя фазовые границы диаграммы, рассчитаны координаты точки Лифщица и области локализации фаз с различными значениями волнового вектора.
\end{abstract}

Ключевые слова: ANNNI-модель, Монте-Карло, алгоритм Ванга-Ландау, модулированное упорядочение.

DOI: $10.21883 /$ FTT.2019.10.48269.461

\section{1. Введение}

В природе помимо обычных видов магнитного упорядочения возникают и некоторые экзотические структуры в виде периодического изменения намагниченности вдоль одного из кристаллографических направлений. Типичным примером такого упорядочения являются длиннопериодические модулированные структуры, возникающие в системах с конкуренцией обменных взаимодействий. В таких системах возможно возникновение и совместное сосуществование нескольких фаз: ферромагнитных, антиферромагнитных, модулированных и парамагнитных. Реальных материалов, в которых реализуются подобные явления, давно уже перевали за сотню [1].

Для описания систем с модулированным магнитным упорядочением используются различные модели. Наиболее простой и эффективной среди них является анизотропная модель Изинга с конкурирующими взаимодействиями со вторыми ближайшими соседями (ANNNI-модель, рис. 1). Отметим, что подобная модель успешно используется для описания термодинамики масляных микроэмульсий и носит название модели Видома [2]. Указанная модель, хотя и не позволяет описать какую либо реальную систему, тем не менее, дает хорошее качественное описание свойств систем с модулированным упорядочением.

При исследовании квазиодномерных и квазидвумерных магнетиков на основе соединений переходных металлов в основном применяются упрощенные многоэлектронные подходы с использованием моделей Гей- зенберга и Изинга [3,4]. Гамильтонианы этих моделей имеют довольно простую алгебраическую структуру. Как показывает практика, основные заключения о свойствах магнитных систем, полученные в рамках этих упрощенных моделей, как правило, не сохраняются и при переходе к более реалистичным объектам, в которых учитываются взаимодействия спинов расположенных вне первой координационной сферы $[1,5]$.

Экспериментальное и теоретическое изучение термодинамических и магнитных свойств является довольно сложной задачей даже для таких простых случаев упорядочения как ферромагнетики или антиферромагнетики. Поэтому в настоящее время наиболее приемлемое описание свойств сложных систем дают только машинные расчеты методами Монте-Карло и молекулярной динамики.

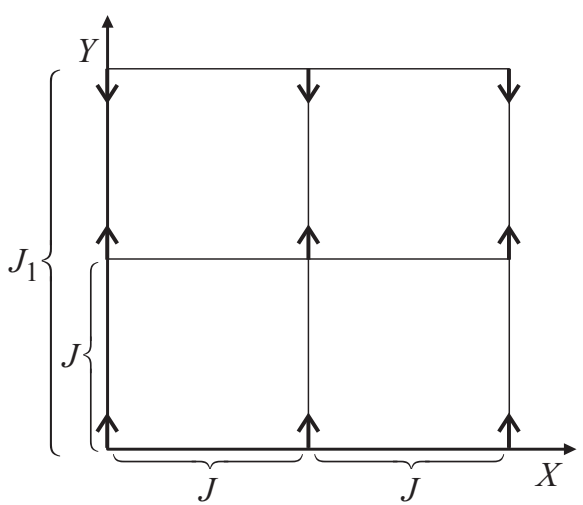

Рис. 1. Двумерная ANNNI-модель. 

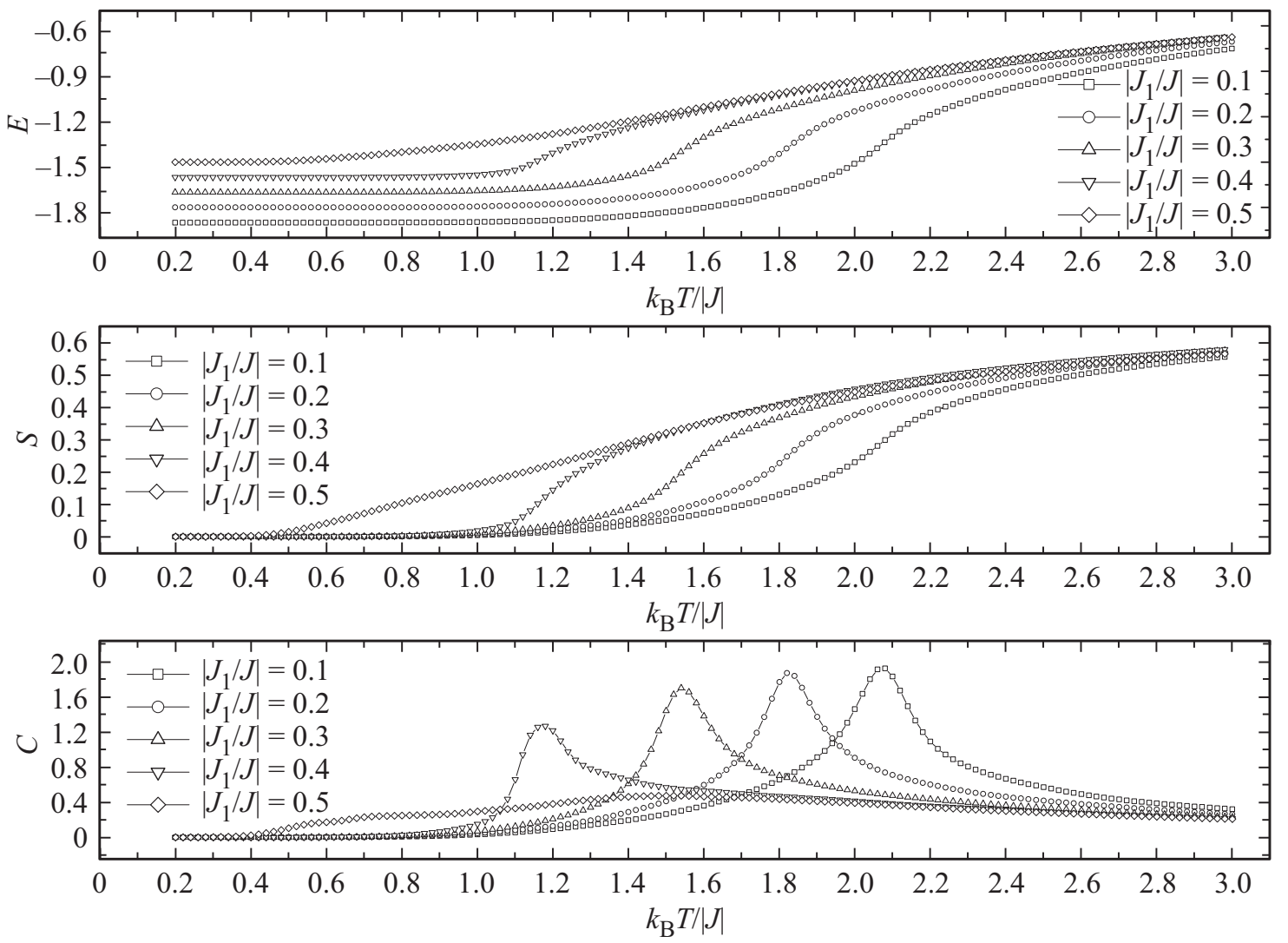

Рис. 2. Температурные зависимости внутренней энергии $E$, энтропии $S$, и теплоемкости $C$ для системы с $L=32$ при $\left|J_{1} / J\right| \leq 0.5$.

В данной работе нами представлены результаты, полученные при исследовании двумерной ANNNI-модели методами Монте-Карло на основе алгоритма Ванга-Ландау представляющие интерес для описания соразмерных и несоразмерных структур возникающих в магнитных материалах.

\section{2. Модель}

Модель Изинга с конкурирующими взаимодействиями (ANNNI-модель) была введена для описания упорядоченных магнитных фаз в кристаллах $\mathrm{CeSb}$ [6].

Гамильтониан модели

$$
H_{\mathrm{ANNNI}}=-J \sum_{i, j} s_{i} s_{j}-J_{1} \sum_{i, j} s_{i} s_{i+2},
$$

где $s_{i}= \pm 1$ спиновая переменная, $J>0$ - параметр обменного взаимодействия соседних пар спинов, $J_{1}<0-$ параметр антиферромагнитного взаимодействия соседей, следующих за ближайшими вдоль оси $Y$.

Рассматриваемая модель исследовалась различными приближенными теоретическими методами. Согласно литературным данным с понижением температуры в ANNNI-модели происходит фазовый переход второго рода из парамагнитного в ближайшее упорядоченное состояние, и переход первого рода из модулированного состояния в ферромагнитную фазу [7].

\section{3. Результаты и их обсуждение}

При проведении исследований на ЭВМ формировались спиновые системы квадратной формы $L \times L$ с размерами $L=32\left(N_{\text {эф }}=L \times L=1024\right)$. Для исключения граничных эффектов на рассматриваемые системы накладывались периодические граничные условия. Моделирование выполнялось при помощи высокоэффективного алгоритма Ванга-Ландау [8]. Использование данного алгоритма позволило получить статическую функцию распределения состояний $G$ и основные спиновые конфигурации системы. Функция распределения играет важную роль при решении задач статистической физики, поскольку она позволяет простым усреднением рассчитать все остальные термодинамические параметры системы. В предыдущей работе [9] приведены полученные нами графики распределения натурального логарифма плотности состояний и соответствующие этим состояниям значения параметра порядка для системы с $L=32$ при значениях $\left|J_{1} / J\right|=0.1 \div 1.0$.

Используя значения $G$ из [9] в данной статье вычислены термодинамические и магнитные параметры системы, усредняя по формуле

$$
A(T)=\frac{1}{Z} \sum_{i=1}^{Q} \frac{A_{i}}{k_{\mathrm{B}} T} \cdot G\left(U_{i}\right) \cdot e^{-U_{i} / k_{\mathrm{B}} T},
$$

где $A_{i}-$ значения вычисляемого параметра (энергия - $U_{i}$ или параметра порядка - $M$ ); 

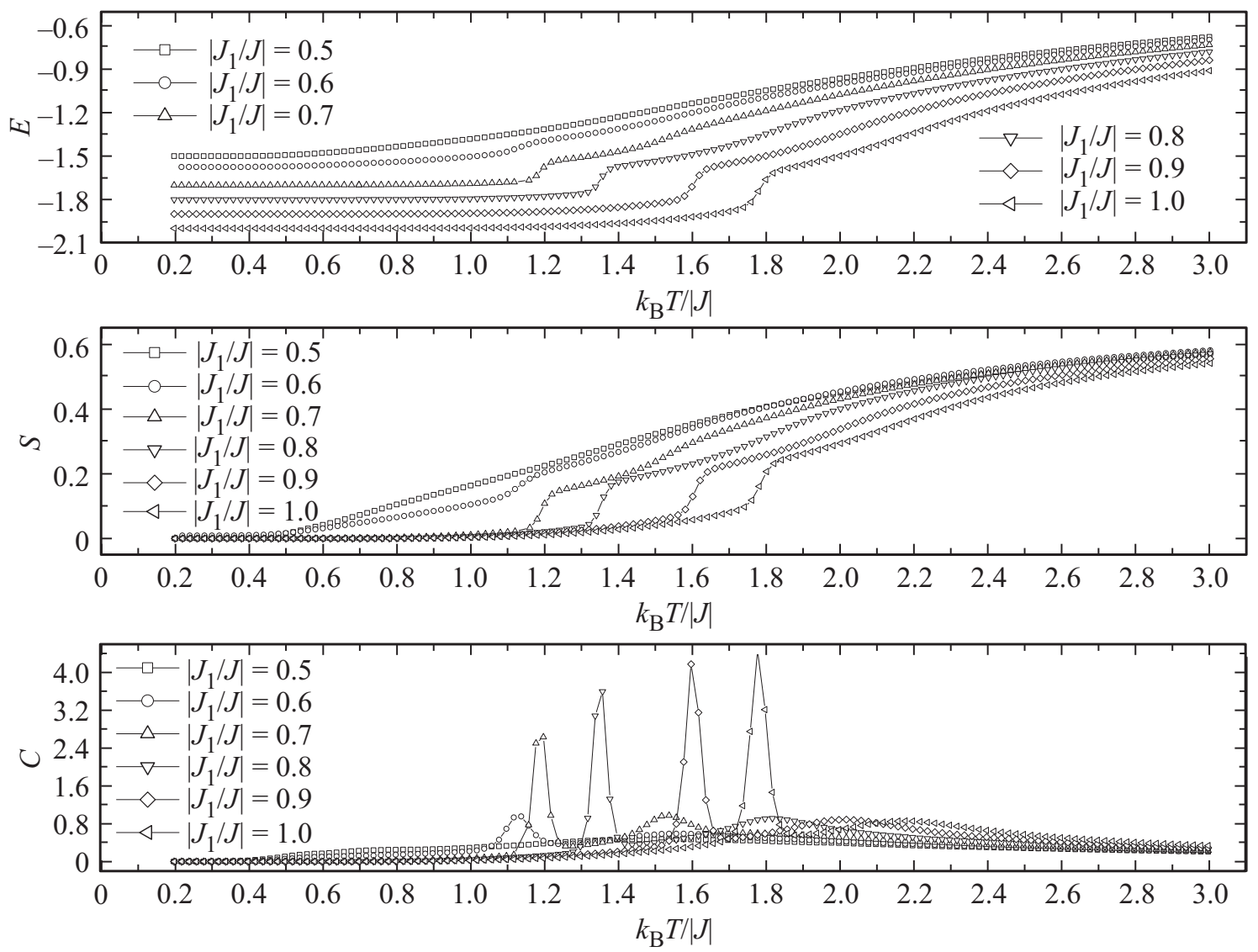

Рис. 3. Температурные зависимости термодинамических параметров для системы с $L=32$ при $\left|J_{1} / J\right| \geq 0.5$.

$Z=\sum_{i=1}^{Q} G\left(U_{i}\right) \cdot e^{-U_{i} / k_{\mathrm{B}} T}-$ статистическая сумма; $Q-$ общее число состояний с различной энергией, $G\left(U_{i}\right)$ - плотность распределения энергии $U_{i}, T-$ температура, $k_{\mathrm{B}}-$ постоянная Больцмана.

Подставляя в формулу (2) в качестве $A_{i}$ значения $U_{i}$ то можно получить графики температурных зависимостей термодинамических параметров (рис. 2-3).

Здесь необходимо отметить тот факт, что характер этих зависимостей сильно зависят от интервала изменения величины конкурирующего взаимодействия. Это достаточно хорошо выражено для двух областей изменения $\left|J_{1} / J\right|$. Так при $\left|J_{1} / J\right|<0.5$ наблюдаем по одному ярко выраженному пику на температурных зависимостях теплоемкости, тогда как при $\left|J_{1} / J\right|>0.5$ таких пиков несколько. Анализ температурных зависимостей термодинамических параметров показывает наличие небольших скачков при $\left|J_{1} / J\right|>0.2$ на графиках внутренней энергии и энтропии для значений температур соответствующих максимуму теплоемкости.

Для значения $\left|J_{1} / J\right|=0.5$ исчезают пики на графиках теплоемкости и скачки на зависимостях внутренней энергии и энтропии. С увеличением значения конкурирующего взаимодействия наблюдается увеличение скачков внутренней энергии и энтропии, а также рост максимального значения теплоемкости. Физической причиной такого поведения является то, что в системе при $\left|J_{1} / J\right|>0.2$ с ростом температуры происходит фазовый переход в сильно вырожденное модулированное состояние.

Аналогично используя в формуле (2) в качестве $A_{i}$ значения $M_{i}$ можно рассчитать температурные зависимости магнитных параметров системы (рис. 4-5).

Как видно из представленных рисунков характер температурных зависимостей магнитных параметров аналогичен тому, что было описано выше для термодинамических свойств.

Поскольку для рассматриваемой модели наиболее интересным является модулированное состояние, то более подробно проводился анализ именно этой области. Для этого был проведен спектральный анализ возникающих структур. Типичные результаты такого анализа для системы с $L=32$ при $T=1.4$ показаны на рис. 6 в виде графика зависимости значений волнового числа $q$ от параметра конкурирующего взаимодействия. Аналогично можно получить области модулированных структур для всей рассматриваемой области. Как видно по рисунку с ростом конкуренции происходит увеличение волнового числа $q$ от нуля до 0.25 . На рисунке стрелками показан характер упорядочения возникающих в исследуемой системе структур.

Обобщая полученные результаты можно построить фазовую диаграмму рассматриваемой системы в координатах $\left|k_{\mathrm{B}} T / J\right|-\left|J_{1} / J\right|$ (рис. 7). На данном рисунке 

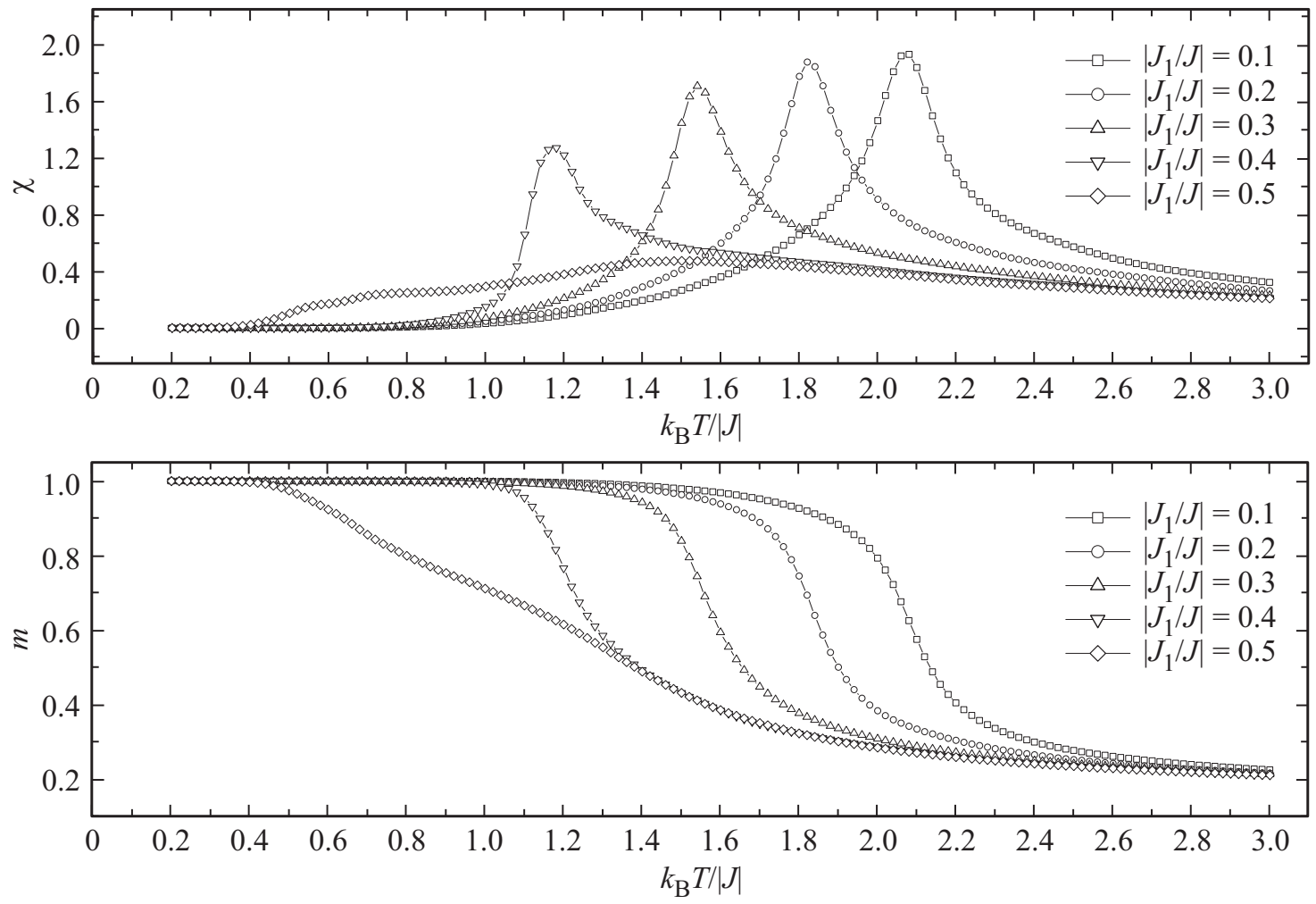

Рис. 4. Температурные зависимости восприимчивости $\chi$ и параметра порядка $m$ для системы с $L=32$ при $\left|J_{1} / J\right| \leq 0.5$.
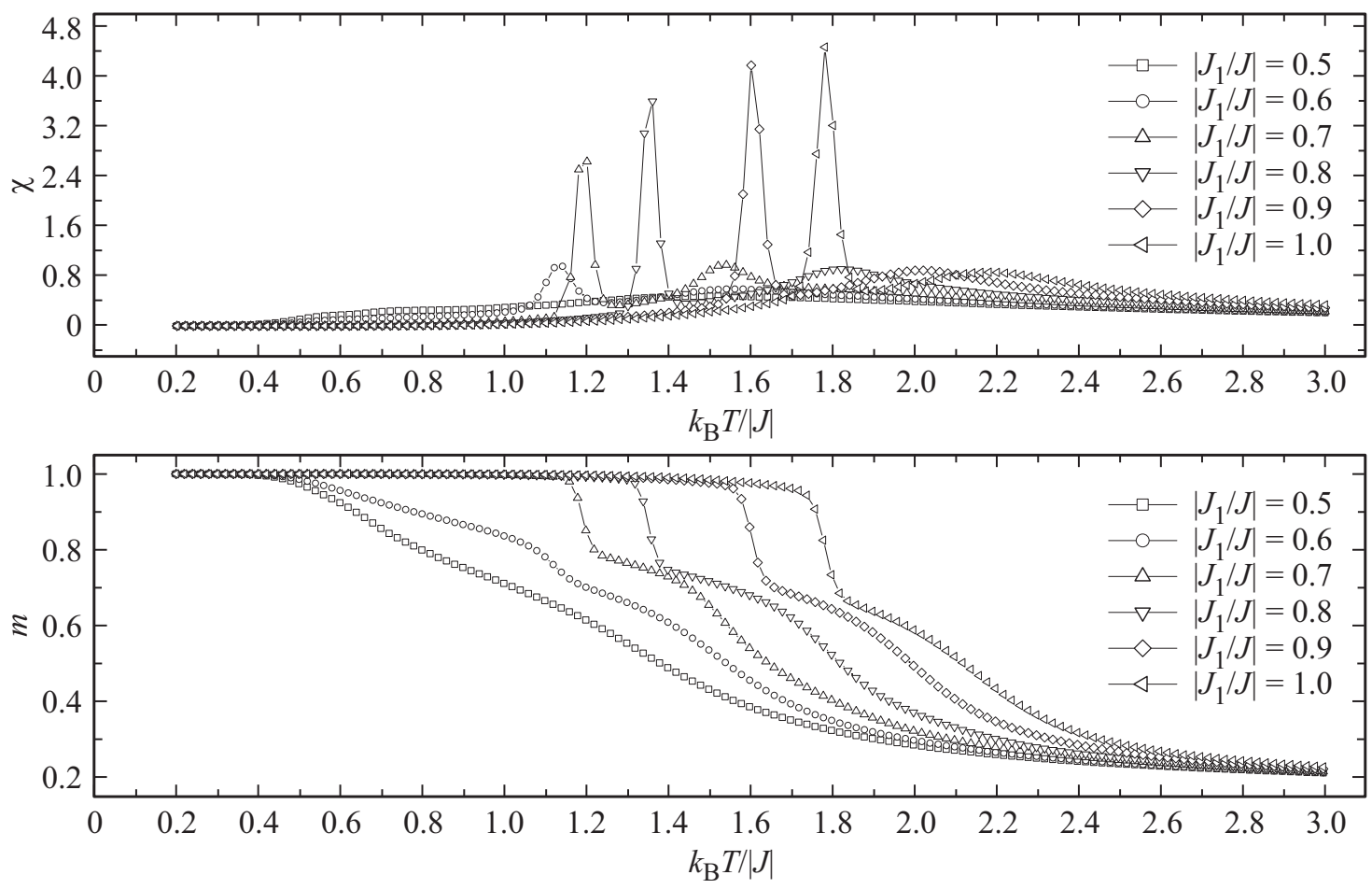

Рис. 5. Температурные зависимости магнитных параметров для системы с $L=32$ при $\left|J_{1} / J\right| \geq 0.5$. 


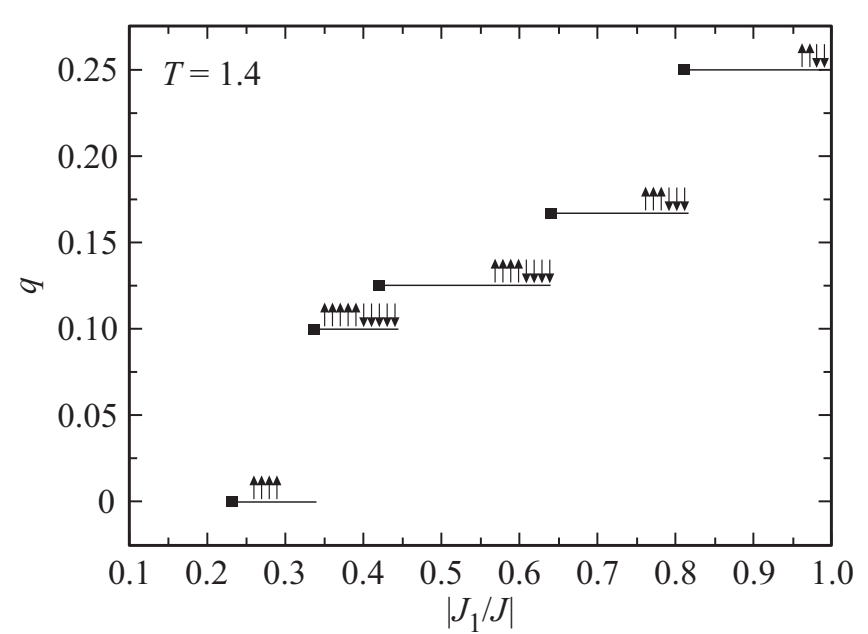

Рис. 6. Зависимость волнового числа от величины конкурирующего взаимодействия (стрелками показан характер магнитного упорядочения).

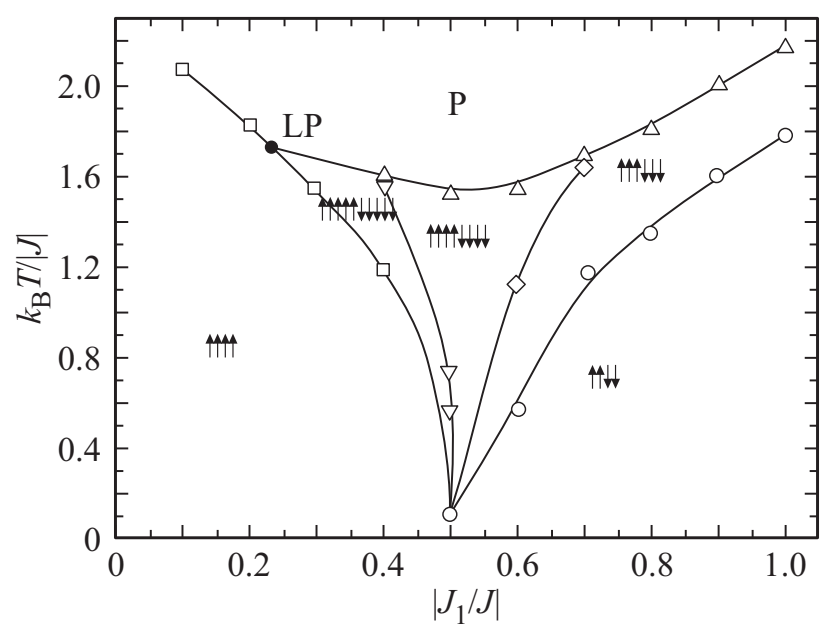

Рис. 7. Фазовая диаграмма двумерной анизотропной модели Изинга с конкурирующими взаимодействиями.

фазовые границы стоились усреднением значений температур соответствующих максимумам теплоемкости и восприимчивости. Интерполируя полученные границы, определяем координаты точки Лифшица $\left(\left|J_{1} / J\right|=0.232\right.$; $\left.\left|k_{\mathrm{B}} T / J\right|=1.733\right)$ и области локализации модулированных структур с различными значениями волнового числа.

Как видно по рис. 7 фазовая диаграмма двумерной анизотропной модели Изинга с конкурирующими взаимодействиями качественно согласуется с диаграммой полученной ранней в других работах и другими теоретическими методами [6]. Диаграмма состоит из четырех больших областей с различными видами магнитного упорядочения. Помимо этого модулированная область разделена на три части в пределах, которых локализованы структуры с различными значениями волнового вектора.

\section{4. Заключение}

По полученным в данной работе результатам можно сделать следующие заключения и выводы. Термодинамика и магнитные свойства двумерной анизотропной модели Изинга с конкурирующими взаимодействиями являются достаточно сложными и интересными. Характерной особенностью рассматриваемой модели можно считать новый тип магнитного упорядочения - длиннопериодические модулированные структуры. Такое упорядочение приводит к появлению в рассматриваемой модели таких экзотических явлений как мультикритическая точка типа Лифшица в которой одновременной сосуществует три фазы: ферромагнитная, модулированная и парамагнитная. Также можно отметить и мультифазную точку при $\left|J_{1} / J\right|=0.5$, которая отделяет области с различным характером поведения термодинамических и магнитных свойств. На основании полученных результатов нам также удалось построить фазовую диаграмму рассматриваемой модели, рассчитать координаты точки Лифшица и определить области локализации модулированных структур с различными значениями волнового числа.

\section{Финансирование работы}

Работа выполнена при поддержке Российского фонда фундаментальных исследований проект № 19-02-00153.

\section{Конфликт интересов}

Авторы заявляют, что у них нет конфликта интересов.

\section{Список литературы}

[1] Ю.А. Изюмов, В.М. Сыромятников. Фазовые переходы и симметрия кристаллов. Наука, М. (1984). 241 с.

[2] B. Widom. J. Chem. Phys. 84, 6943 (1986).

[3] А.А. Овчинников, И.И. Украинский, Г.Ф. Квенцель. УФН 108, 81 (1972).

[4] S.R. White. Phys. Rep. 301, 187 (1998).

[5] M.A. Shakirov, V.S. Tsibulsky, A.E. Antipov, Y.E. Shchadilova, A.N. Rubtsov. Sci. Rep. 5, 8005 (2015).

[6] R.J. Elliott. Phys. Rev. 124, 346 (1961).

[7] A.K. Murtazaev, J.G. Ibaev. Solid State Commun. 152, 177 (2012).

[8] F. Wang, D.P. Landau. Phys. Rev. Lett. 86, 2050 (2001).

[9] А.К. Муртазаев, Ж.Г. Ибаев. Вестн. ДГУ. Сер. Естеств. науки 33, 40 (2018).

Редактор К.В. Емцев 